Reprod. Nutr. Dévelop., 1988, 28 (2 A), 241-252.

\title{
Caractérisation et évolution physiologique des récepteurs pour les " insulin-like growth factors " I et II (IGFs) dans la glande mammaire de brebis
}

\author{
Catherine DISENHAUS, Lucette BELAIR, J. DJIANE
}

Laboratoire de Physiologie de la Lactation, I.N.R.A., 78350 Jouy-en-Josas, France.

Summary. Characterization and physiological variations of /GFs receptors in the ewe mammary gland.

Membrane preparations were obtained by differential centrifugations of ewe mammary gland homogenates. These membrane preparations contained specific receptors for IGF and $I_{G F}$ which possess high affinities for their specific ligands ( $\mathrm{Ka} .5$ to $1.510^{9} \mathrm{M}^{-1}$ ).

Maximum binding of ${ }^{125} \mid \mathrm{IGF}_{1}$ was obtained after $48 \mathrm{~h}$ at $4{ }^{\circ} \mathrm{C}$. This binding was inhibited by unlabelled $I \mathrm{GF}_{1}(\mathrm{ED} 50=14 \mathrm{ng} / \mathrm{ml})$, partially inhibited by high concentrations of insulin $(50 \mu \mathrm{g} / \mathrm{ml})$. Prolactin (oPRL), growth hormone (bGH) or relaxine (Rel) were without effect.

Maximum binding of ${ }^{125} \mid \mathrm{IGF}_{2}$ was obtained after $6 \mathrm{~h}$ at $20^{\circ} \mathrm{C}$. This binding was inhibited by unlabelled $\mathrm{IGF}_{2}(\mathrm{ED} 50=44 \mathrm{ng} / \mathrm{ml})$, partially inhibited by $\mathrm{IGF}_{1}(\mathrm{ED} 50=$ $200 \mathrm{ng} / \mathrm{ml}$ ) and unmodified by INS, PRL, bGH or Rel.

Receptor numbers for $I G F_{1}$ were significantly higher $(p<0.01)$ on day 100 of pregnancy $(N=480 \pm 17$ fmoles $/ \mathrm{mg}$ proteins) compared to day 20 of lactation ( $\mathrm{N}=174 \pm 21 \mathrm{fmoles} / \mathrm{mg}$ ).

The numbers of $I_{G F}$ receptors were always higher than those of IGF $_{1}$ receptors. During pregnancy the numbers of $I G F_{2}$ receptors $(N=1860 \pm 157 \mathrm{fmoles} / \mathrm{mg})$ were also higher than during lactation.

These results suggest that the mammary gland may constitute a target organ for IGFs. These factors could be involved in the regulation of mammary gland development and during cell differentiation.

\section{Introduction.}

L'importance de l'hormone de croissance $(\mathrm{GH})$ dans le complexe hormonal lactogène chez les ruminants a été soulignée depuis de nombreuses années (Cowie et Tindal, 1960 ; Denamur, 1971). II est maintenant bien connu que l'administration de $\mathrm{GH}$ ou de son facteur de libération hypothalamique (GRF) augmente significativement la production laitière (Enright et al., 1986 ; Hart et al., 1985).

Néanmoins, la $\mathrm{GH}$ bovine ( $\mathrm{bGH}$ ) n'augmente pas la biosynthèse des caséines ou des matières grasses, ni la sécrétion $d^{\prime} \alpha$-lactalbumine d'explants mammaires bovins maintenus en culture (Gertler, Cohen et Maoz, 1983). De plus, il n'a pas été mis en évidence de récepteurs pour la $\mathrm{GH}$ dans le tissu mammaire (Gertler, Ashkenazi et Madar, 1984 ; Keys et Djiane, 1987). 
L'action de la GH sur la glande mammaire pourrait donc être médiée, soit par des substances dont la libération est dépendante de la $\mathrm{GH}$, soit par un changement dans la partition des nutriments, soit par les deux.

Les somatomédines (" insulin-like growth factors ": IGFs) sont des maillons de l'axe somatotrope qui constituent une famille d'hormones peptidiques impliquée dans diverses activités métaboliques telles que le transport de glucose et d'acides aminés, la synthèse protéique, la synthèse d'ADN et d'ARN et la multiplication cellulaire (Van Wyk et al., 1974). Les deux types d'IGFs du sér um humain - IGF $F_{1}$ et $\mathrm{IGF}_{2}$ - ont été isolés et les structures primaires déterminées par Rinderknecht et Humbel (1978). La structure des IGFs semble très conservée lors de l'évolution (Honegger et Humbel, 1986). L'IGF 1 bovin est identique à I'IGF humain. Seuls 3 acides aminés différencient $I^{\prime} I G F_{2}$ bovin de I'IGF $F_{2}$ humain. Cette différence structurale est suffisante pour que le blGF $\mathrm{G}_{2}$ montre moins de $10 \%$ de réactivité immunologique croisée avec le $\mathrm{h}-\mathrm{IGF}_{2}$.

Deux différents types de récepteurs pour les IGFs ont été identifiés : un plus

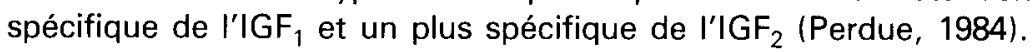

II faut noter la très large distribution des récepteurs IGFs dans l'organisme : de multiples organes dans différentes espèces possèdent des récepteurs pour l'un ou les deux facteurs (Rosenfeld et Hintz, 1986). Aucune publication ne rapporte l'existence de récepteurs pour les IGFs dans la glande mammaire normale. Le récepteur de type $\mathrm{I}$, usuellement considéré comme étant le récepteur pour I'IGF est structurellement très proche du récepteur insulinique mais en diffère par sa moindre réactivité à l'anticorps anti-récepteur insulinique et surtout par l'affinité relative des différents ligands: I'insuline se lie au récepteur de type I mais avec une affinité très inférieure à $I^{\prime} I G F_{1}$ et à $I^{\prime} \mid G F_{2}$. Le récepteur de type $\|$ a une affinité supérieure pour $I \mathrm{GF}_{2}$ que pour $\mathrm{IGF}_{1}$ et $\mathrm{n}^{\prime}$ a pas d'affinité pour l'insuline.

L'ensemble de ces données: I'action de la $\mathrm{GH}$ sur la production laitière, l'absence de récepteurs pour la GH dans le tissu mammaire et la très large distribution des récepteurs pour les IGFs chez les mammifères - nous ont conduit à rechercher la présence de récepteurs pour les IGFs dans la glande mammaire.

II sera décrit d'une part, les caractéristiques des liaisons spécifiques des ${ }^{125}$ |$I \mathrm{GF}_{1}$ et ${ }^{125} \mathrm{IIGF}_{2}$ et, d'autre part, l'évolution des récepteurs au cours du cycle gestation-lactation chez la brebis.

\section{Matériels et méthodes.}

Animaux. - Des brebis de race Préalpes du Sud ont été sacrifiées à diverses périodes de gestation et de lactation. Les glandes mammaires ont été prélevées, disséquées, pesées et conservées à $-20^{\circ} \mathrm{C}$.

Préparations membranaires. - Le tissu mammaire, dégraissé autant que faire se peut, a été découpé en petits fragments puis homogénéisé à l'aide d'un broyeur " Polytron » dans un tampon Tris $(25 \mathrm{mM}) \mathrm{pH} 7,5$ contenant $10 \mathrm{mM}$ de $\mathrm{MgCl}_{2}$ et $1 \mathrm{mM}$ de phénylméthylsulfonylfluoride (PMSF, Sigma), à raison de $3 \mathrm{ml}$ de tam- 
pon par gramme de tissu. L'homogénat est filtré à travers deux épaisseurs de gaze et centrifugé une première fois à $4000 \mathrm{~g}$ pendant $20 \mathrm{~min}$ à $4{ }^{\circ} \mathrm{C}$.

Le culot est éliminé et le surnageant est de nouveau centrifugé à $100000 \mathrm{~g}$ pendant $90 \mathrm{~min}$. Le culot de cette deuxième centrifugation est repris dans le même tampon Tris, homogénéisé dans un Potter téflon-verre. La concentration protéique de la préparation est déterminée par la méthode de Lowry (1951).

\section{Hormones.}

- L'IGF 1 et I'IGF $F_{2}$ humains purifiés ont été gracieusement fournis par le Pr. Humbel (Zurich). Les hormones sont marquées à l'iode 125 par la méthode à la chloramine $T$ (Hunter et Greenwood, 1962). Les hormones marquées sont séparées de l'iode libre par chromatographie sur colonne Séphadex G25. La radioactivité spécifique obtenue entre 80 et $200 \mu \mathrm{Ci} / \mu \mathrm{g}$.

- Les autres hormones utilisées sont les suivantes : insuline bovine (INS ; Sigma, 25,6 UI/mg), prolactine ovine (oPRL; NIAMDD-oPRL $14: 31 \mathrm{UI} / \mathrm{mg}$ ), hormone de croissance bovine (bGH; NIAMDD-bGH-B $;$; AFP 5200 ) et la relaxine porcine.

\section{Etude des récepteurs.}

- Les protéines membranaires (100 à $400 \mu \mathrm{g}$ ) sont incubées dans un tampon Tris $(25 \mathrm{mM}, \mathrm{pH} 7,5)$ contenant $10 \mathrm{mM}$ de $\mathrm{MgCl}_{2}, 0,1 \%$ de BSA et $0,02 \%$ d'azide de sodium, en présence d'environ $10^{5} \mathrm{cpm}$ d'hormone $\left(I G F_{1}\right.$ ou $\left.\mid G_{2}\right)$ radio-iodée et en absence (liaison totale) ou en présence d'un excès $(1 \mu \mathrm{g})$ d'hormone non radio-iodée (liaison non spécifique). Le volume final de l'incubation est de $500 \mu \mathrm{l}$. L'incubation est arrêtée après $16 \mathrm{~h}$ à $4{ }^{\circ} \mathrm{C}$ dans le cas de $\mathrm{I}^{\prime} \mathrm{IGF}_{1}$ ou après $4 \mathrm{~h}$ à température ambiante pour $\mathrm{I}^{\prime} \mid \mathrm{GF}_{2}$ par addition de $3 \mathrm{ml}$ de tampon d'incubation froid. Les tubes sont centrifugés $(4000 \mathrm{~g})$ pendant $15 \mathrm{~min}$ à $4{ }^{\circ} \mathrm{C}$, le surnageant est éliminé et la radioactivité du culot mesurée dans un compteur à scintillation (LKB). Les résultats sont exprimés en pourcentages de la radioactivité totale spécifiquement liée.

- Le nombre de sites par mg de protéines et l'affinité de la liaison hormonerécepteur ont été calculés selon la méthode de Scatchard (1949) après incubation des protéines membranaires en présence d'environ $10^{5} \mathrm{cpm} \mathrm{d}^{\prime 125}|-| \mathrm{GF}\left(\mid \mathrm{GF} \mathrm{F}_{1}\right.$ ou $I G F_{2}$ ) et de quantités croissantes d'hormone non radio-iodée (IGF $F_{1}$ ou $\left.I_{G F}\right)$.

- Pour l'étude de la spécificité des récepteurs, la liaison du traceur radioactif $\left({ }^{125}|-| G F_{1}\right.$ ou $\left.{ }^{125}|-| G_{2}\right)$ sur des préparations membranaires est mise en compétition avec des concentrations croissantes des différentes préparations hormonales $\left(I G F_{1}, I G F_{2}\right.$, insuline, bGH, relaxine, prolactine). Les résultats sont exprimés en pourcentage de la liaison observée en l'absence de compétiteur. 
Résultats.

Caractérisation des récepteurs pour les /GFs dans la glande mammaire.

- Cinétique de liaison des ${ }^{125 /-/ G F s ~ e n ~ f o n c t i o n ~ d e ~ l a ~ t e m p e ́ r a t u r e . ~-~ T r o i s ~}$ températures usuelles ont été étudiées : $4{ }^{\circ} \mathrm{C}, 20^{\circ} \mathrm{C}$ (température ambiante) et $37{ }^{\circ} \mathrm{C}$ pendant différentes durées d'incubation.

Les résultats obtenus sont lès suivants: le maximum de la liaison de I-IGF est obtenu après $48 \mathrm{~h}$ à $4^{\circ} \mathrm{C}$. Environ $75 \%$ de cette liaison maximale sont obtenus après $16 \mathrm{~h}$. A $20^{\circ} \mathrm{C}$, la liaison de |'125/-IGF 1 est moindre et à $37^{\circ} \mathrm{C}$ cette liaison n'est pas stable (fig. 1A). La dissociation de la liaison est difficile à réaliser par
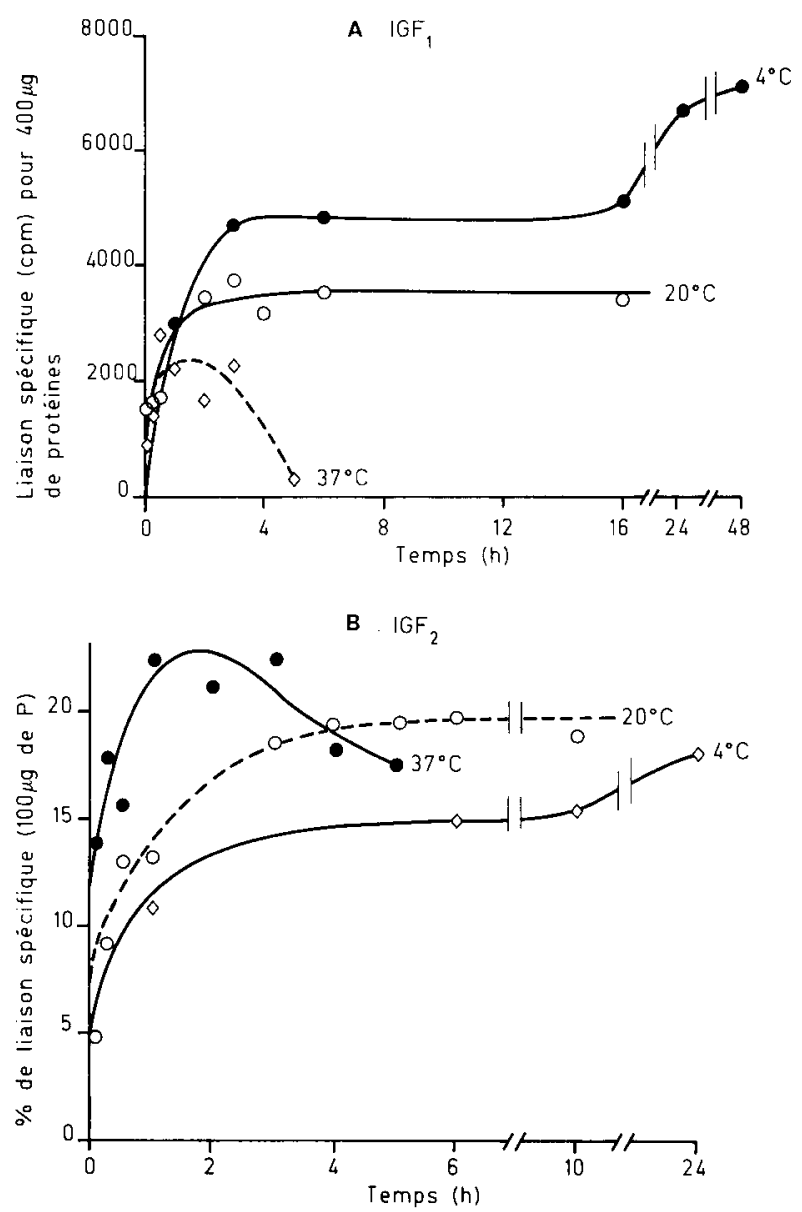

FIG. 1. - Cinétique de liaison des $125 /-/ G F s$ à différentes températures $\left(4^{\circ} \mathrm{C}, 20^{\circ} \mathrm{C}, 37^{\circ} \mathrm{C}\right)$. A : $400 \mu \mathrm{g}$ de protéines membranaires ont été incubés en présence d'environ $10^{5} \mathrm{cpm}^{1}{ }^{125} I_{\mathrm{GF}}$. $\mathrm{B}$ : $100 \mu \mathrm{g}$ de protéines membranaires ont été incubés en présence d'environ $10^{5} \mathrm{cpm} \mathrm{d}^{\prime 125} \mathrm{IGF}_{2}$. 
simple dilution du traceur mais est obtenue par l'addition d'une forte concentration d'insuline accompagnant la dilution (fig. 2A). La figure $1 \mathrm{~B}$ montre la cinétique de liaison de $I^{\prime 125} \mid-\mathrm{IGF}_{2}$ à différentes températures. A $37^{\circ} \mathrm{C}$, la liaison est importante mais instable. A $20^{\circ} \mathrm{C}$, le maximum de la liaison est obtenu après $6 \mathrm{~h}$. Dès $4 \mathrm{~h}$ d'incubation, plus de $95 \%$ de la liaison maximale sont observés. A $4{ }^{\circ} \mathrm{C}$, la liaison du traceur est plus lente et moins importante. La dissociation ne se réalise pas par dilution du traceur (fig. 2B).

- Spécificité de la liaison des $125 /-/ G F s$. - Comme le montre la figure 3A, la liaison de $I^{\prime 125}|-| G F_{1}$ est inhibée par $I^{\prime} \mid G_{1} F_{1}$ non radioactif $(D E 50 \simeq 14 \mathrm{ng} / \mathrm{ml}$ ), partiellement inhibée par $\mathrm{I}^{\prime} \mathrm{IGF}_{2}$ et par de fortes concentrations d'insuline $(50 \mu \mathrm{g} / \mathrm{ml})$. De même, la liaison de $I^{\prime 125} \mid-I_{G F}$ est inhibée par $I^{\prime} \mid G_{2}$ non radioactive $(D E 50 \simeq 20 \mathrm{ng} / \mathrm{ml}$ ) (fig. 3B), partiellement inhibée par I'IGF (DE $50 \simeq 150 \mathrm{ng} / \mathrm{ml}$ ). Même de fortes concentrations d'insuline n'ont aucun effet sur la liaison de $\mathrm{I}^{\prime 125} \mathrm{I}_{\mathrm{IGF}}$.

Les liaisons des ${ }^{125}$ I-IGFs ne sont pas modifiées par l'addition des autres hormones utilisées.

- Affinité de la liaison. - La figure 4 montre un exemple d'analyse du nombre et de l'affinité des récepteurs selon la méthode de Scatchard pour chacune des deux hormones dans des préparations membranaires de glande mammaire en lactation. Comme nous le verrons plus loin, le nombre de sites récepteurs varie en fonction du stade physiologique. Cependant, il apparaît nettement que les préparations membranaires de glandes mammaires contiennent des récepteurs de haute affinité ( $\mathrm{Ka}$ compris entre 0,4 et $1,510^{9} \mathrm{M}^{-1}$ ) pour les somatomédines.
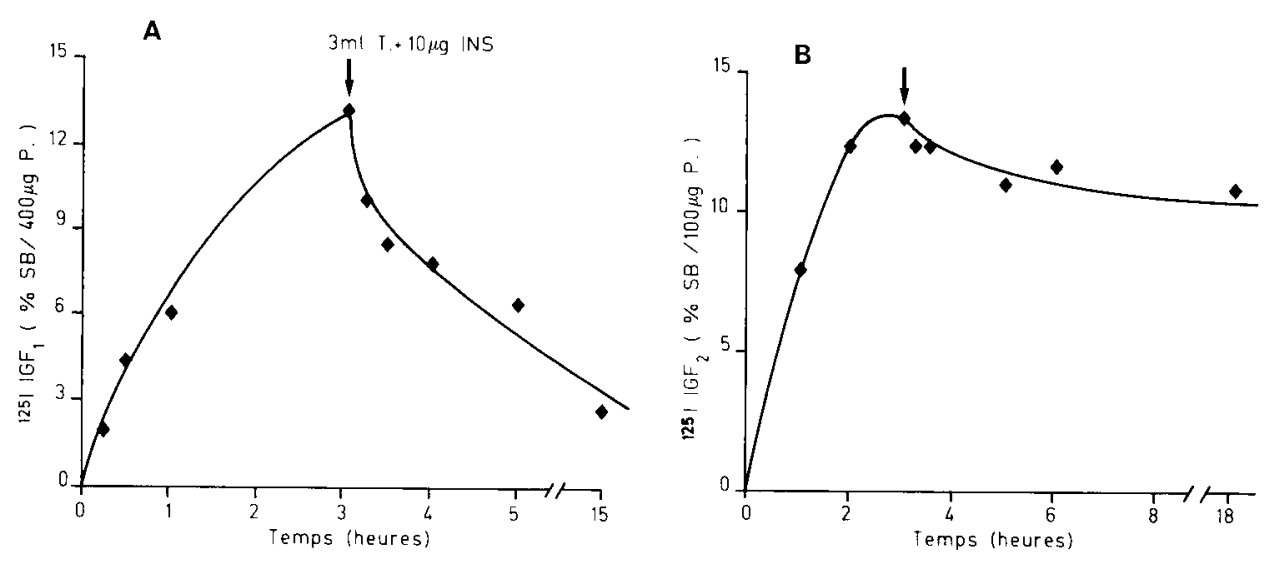

FIG. 2. - Cinétique d'association et de dissociation des ${ }^{125} /-/ G F s$. A : IGF 1 : I'association est arrêtée par addition de $3 \mathrm{ml}$ de tampon d'incubation froid $\left(4^{\circ} \mathrm{C}\right)$ et de $10 \mu \mathrm{g}$ d'insuline après $3 \mathrm{~h}$ à $20^{\circ} \mathrm{C}$. $\mathrm{B}$ : $\mid \mathrm{GF}_{2}$ : I'association est arrêtée par addition de $3 \mathrm{ml}$ de tampon d'incubation froid $\left(4^{\circ} \mathrm{C}\right)$. 

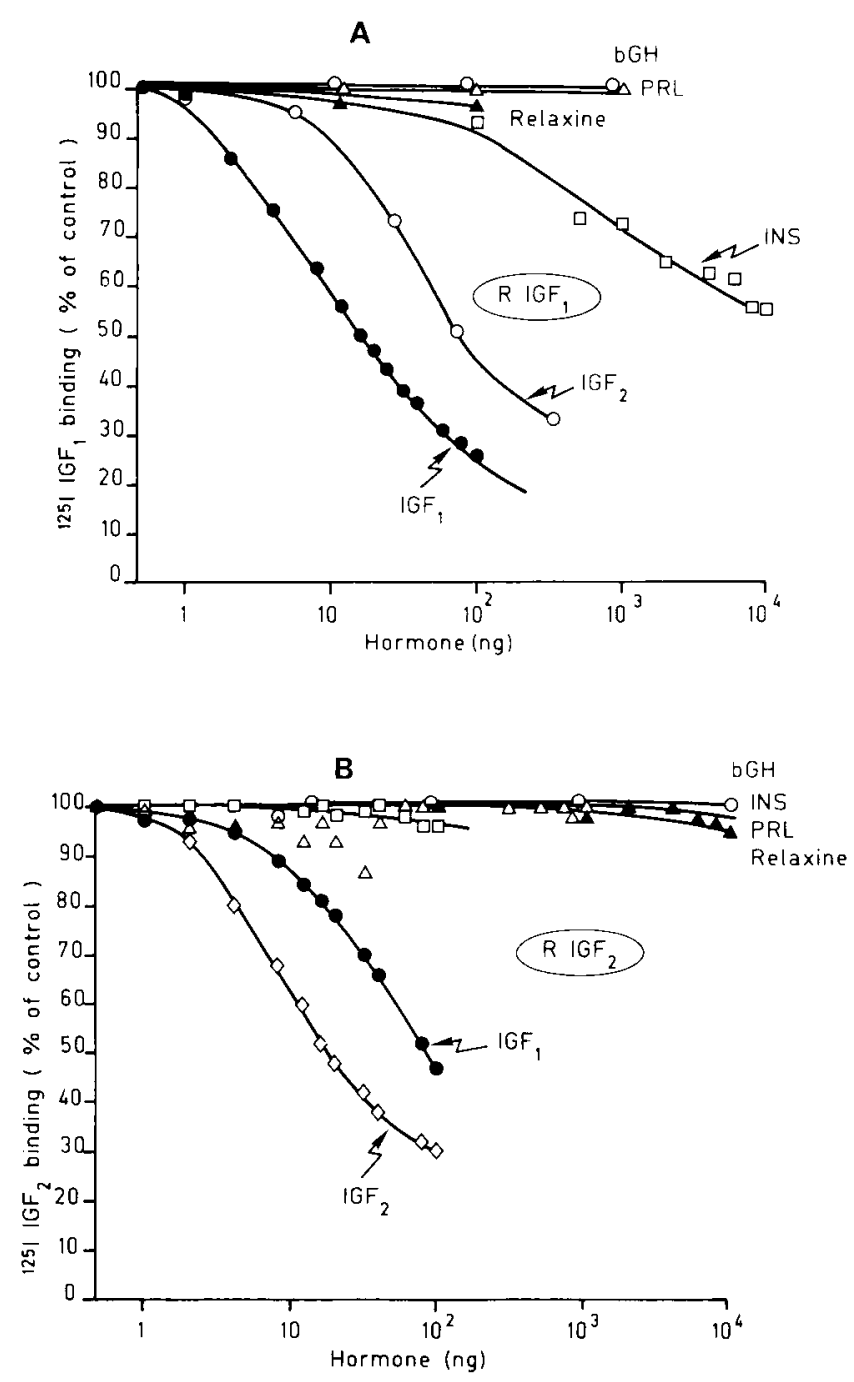

FIG. 3. - Spécificité hormonale de la liaison de $l^{\prime 225} / G F_{1}(A)$ et de $l^{125} / G F_{2}(B)$ sur des préparations membranaires de glandes mammaires. A : I'incubation a été réalisée avec $400 \mu \mathrm{g}$ de protéines membranaires et $10^{5} \mathrm{cpm} \mathrm{d} \mathrm{d}^{\prime 25} \mathrm{IGF}_{1}$ pendant $16 \mathrm{~h}$ à $4^{\circ} \mathrm{C}$. B : I'incubation a été réalisée avec $100 \mu \mathrm{g}$ de protéines membranaires et $10^{5} \mathrm{cpm} \mathrm{d}$ d $^{125} \mid \mathrm{GF}_{2}$ pendant $4 \mathrm{~h}$ à $20^{\circ} \mathrm{C}$.

Au même stade physiologique (20 jours de lactation), les sites récepteurs pour $\mathrm{I}^{\prime} I G F_{2}$ sont 4 à 5 fois plus concentrés que ceux pour l'IGF $F_{1}$. La linéarité des représentations graphiques du calcul selon la méthode de Scatchard indique la mise en évidence d'un seul type de sites récepteurs pour chacune des deux hormones. 

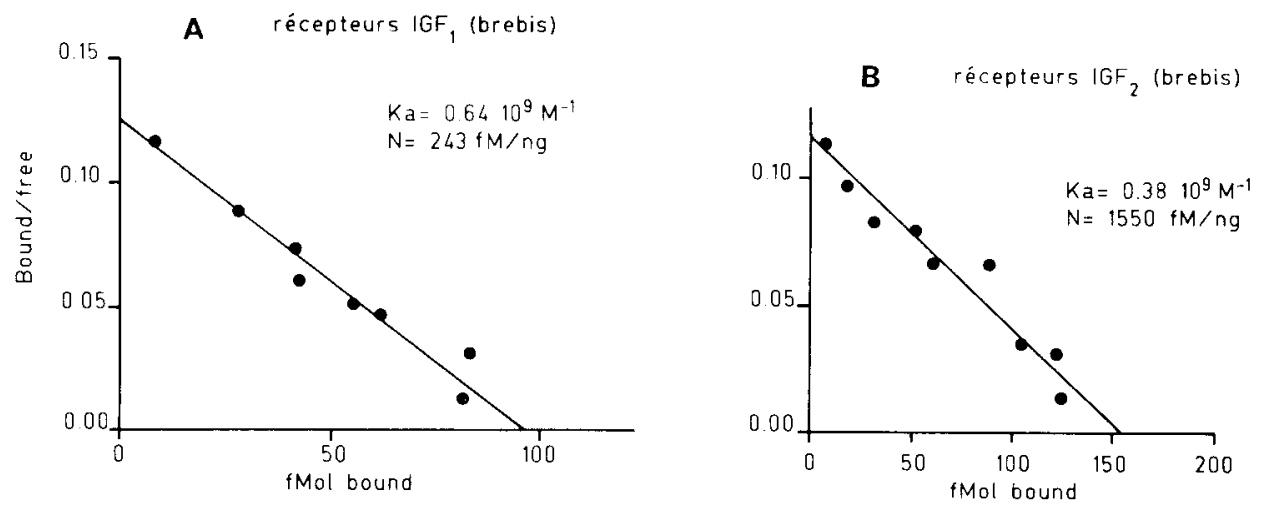

FIG. 4. - Analyse par la méthode de Scatchard de la fixation des ${ }^{125} / G F s$ sur des préparations de glandes mammaires de brebis à 20 jours de lactation. A : $400 \mu \mathrm{g}$ de protéines membranaires ont été incubés pendant $16 \mathrm{~h}$ à $4^{\circ} \mathrm{C}$ en présence de $10^{5} \mathrm{cpm} \mathrm{d}^{\prime 25} \mathrm{IGF}_{\mathrm{L}}$. $\mathrm{B}: 100 \mu \mathrm{g}$ de protéines membranaires ont été incubés pendant $4 \mathrm{~h}$ à $20^{\circ} \mathrm{C}$ en présence de $10^{5} \mathrm{cpm} \mathrm{d}^{\prime 125^{\circ}} \mid \mathrm{GF}_{2}$.

Evolution physiologique du nombre de récepteurs pour les IGFs.

La figure 5 visualise les valeurs individuelles des pourcentages de liaisons spécifiques obtenues avec ${ }^{125} /-\mathrm{IGF}_{1}(\mathrm{~A})$ et ${ }^{125} / \mathrm{IGF} \mathrm{F}_{2}(\mathrm{~B})$ en fonction des différents stades physiologiques.

Trois constatations s'imposent :

- I'observation d'une liaison plus importante de $\left.\right|^{\prime 25|-| G F_{2}}$ par rapport à |'125|$I F_{1}$, quel que soit le stade physiologique envisagé ;

- la grande variabilité individuelle du pourcentage de liaison pendant la gestation. Cette variabilité n'est plus observée à 20 jours de lactation :

- l'observation d'une liaison plus faible à 20 jours de lactation que lors des différents stades de gestation, notamment pour ${ }^{125} \mid-\mathrm{IGF}_{1}$. L'incubation de préparations membranaires en présence des quantités croissantes d'IGF non radioactif a permis de calculer le nombre de récepteurs et l'affinité de la liaison (analyse par la méthode de Scatchard) à 100 jours de gestation et à 20 jours de lactation.

Les résultats sont visualisés par la figure 6.

Si l'affinité de la liaison n'est pas significativement modifiée, le nombre de sites récepteurs pour I'IGF ${ }_{1}$ est significativement plus élevé $(p<0,01)$ à 100 jours de gestation ( $\mathrm{N}=480 \pm 17 \mathrm{fmoles} / \mathrm{mg}$ de protéines) qu'à 20 jours de lactation ( $\mathrm{N}=174 \pm 21 \mathrm{fmoles} / \mathrm{mg}$ de protéines). La même évolution est observée pour les récepteurs $I G F_{2}$ mais avec une moindre amplitude : à 100 jours de gestation les récepteurs sont un peu plus concentrés ( $N=1860 \mathrm{fmoles} \pm 157 / \mathrm{mg}$ ) de protéines) qu'à 20 jours de lactation ( $N=1052$ fmoles $\pm 221 \mathrm{mg}$ de protéines) $(p<0,05)$. 

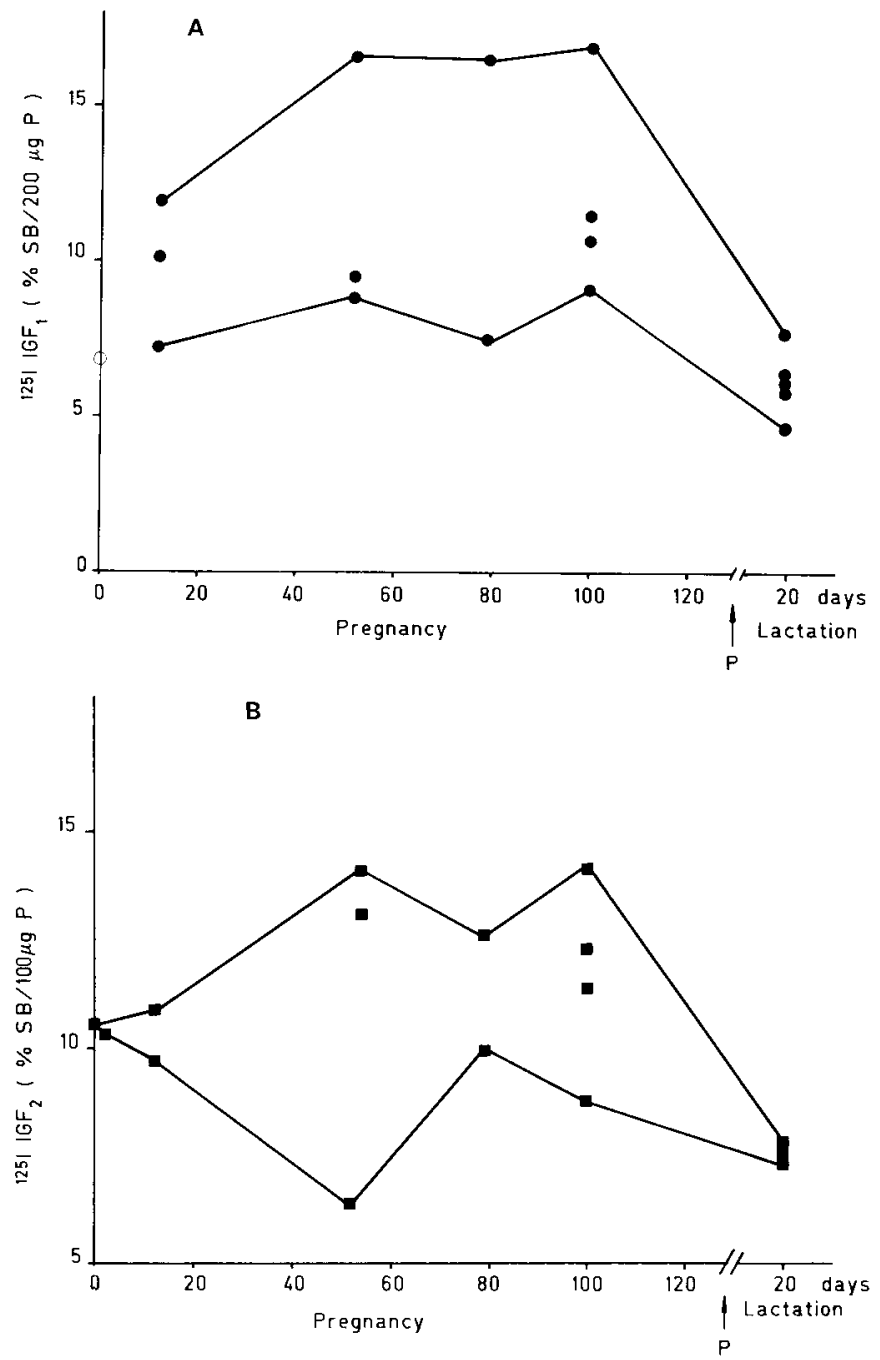

FIG. 5. - Valeurs individuelles des liaisons spécifiques (\%) obtenues par incubation de $l^{125} / G F_{1}$ et de $I^{\prime 25} / G F_{2}(B)$ avec des préparations membranaires de glandes mammaires à différents stades de gestation et de lactation.

Chaque point correspond à la valeur obtenue avec une préparation membranaire d'un animal. La courbe supérieure relie les valeurs maximales par stade, la courbe inférieure les valeurs minimales.

\section{Discussion et conclusion.}

Cette publication décrit la mise en évidence et la caractérisation des récepteurs spécifiques et de haute affinité pour les IGFs dans les préparations membranaires de glande mammaire de brebis. L'analyse des courbes de spécificité (fig. 3) 
A IGF, RECEPTORS IN EWE MAMMARY GLAND

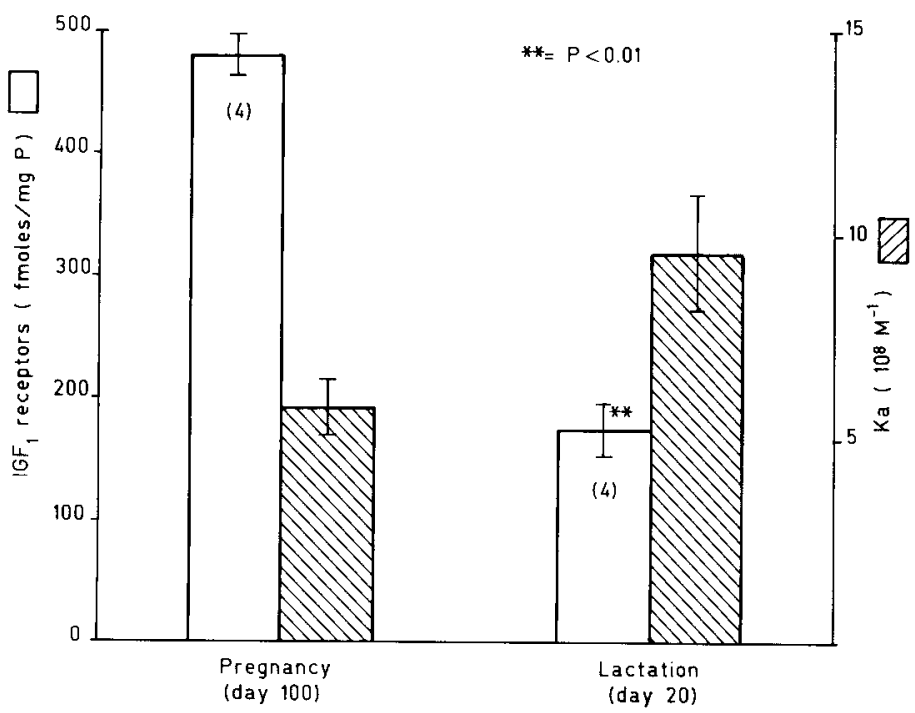

B IGF ${ }_{2}$ IN EWE MAMMARY GLAND

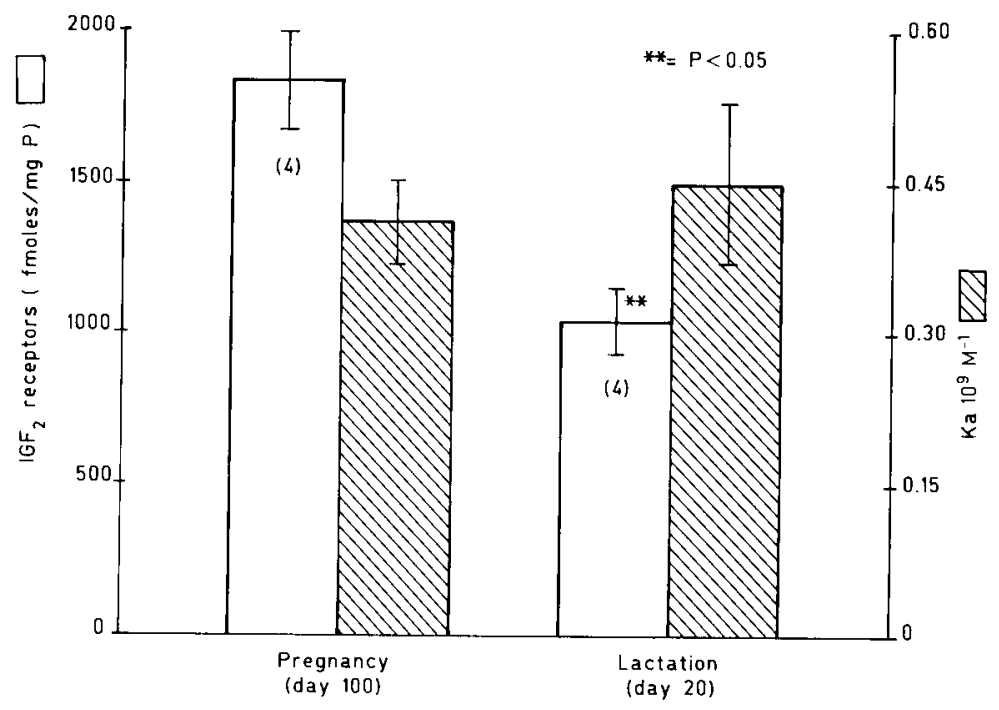

FIG. 6. - Comparaison du nombre de sites récepteurs et de l'affinité de la liaison des $125 / 1 G F S$ $\left(A: I G F_{1} ; B: I G F_{2}\right)$ à 100 jours de gestation et à 20 jours de lactation.

Les résultats représentent la moyenne de 4 animaux par stade et l'erreur standard moyenne correspondante. 
permet d'établir que les deux types de récepteurs pour les IGFs décrits dans d'autres modèles (Rosenfeld et Hintz, 1986) coexistent dans le tissu mammaire ; les récepteurs de type II étant plus concentrés.

Ces résultats indiquent que la cellule mammaire constitue une cible biologique potentielle pour les IGFs. II a été montré sur un type cellulaire tumoral humain (T47D) que I'IGF 1 était capable d'activer la multiplication cellulaire (Furnaletto et Di Carlo, 1984), les effets biologiques des IGFs sur la cellule mammaire normale restent actuellement très peu connus.

Dans les fibroblastes cutanés humains en culture, l'insuline emprunte le récepteur de I'IGF 1 pour stimuler la synthèse d'ADN (Van Wyck et al., 1985 ; Flier et al., 1986). L'hypothèse selon laquelle l'action mitogène de l'insuline se ferait par la même voie dans le tissu mammaire est vraisemblable, compte tenu de l'analyse de la spécificité de liaison que nous avons décrite (fig. 3).

Par ailleurs, il est établi depuis longtemps que la stimulation de la biosynthèse des caséines par la prolactine est considérablement amplifiée par l'insuline. II reste encore toutefois une incertitude sur la nature du récepteur hormonal utilisé par I'insuline dans cette action. En effet, les concentrations actives in vitro (de l'ordre du $\mu \mathrm{g}$ ) sont, d'une part, incompatibles avec celles rencontrées dans le sérum et, d'autre part, compatibles avec une interaction avec les récepteurs de I'IGF (Bolander et al., 1981).

L'étude de l'évolution physiologique du nombre de récepteur pour les IGFs fait apparaître que ceux-ci sont plus nombreux au cours de la gestation qu'à 20 jours de lactation (fig. 6). Cette évolution est particulièrement nette pour les récepteurs d'IGF $F_{1}$ sans qu'on observe de modification significative de l'affinité de la liaison de l'hormone à son récepteur. En accord avec ces résultats, I'IGF 1 pourrait avoir une action propre sur le développement de la glande mammaire en synergie avec la prolactine. Le déterminisme hormonal des variations du nombre de récepteurs reste inconnu.

Cette variation pour les IGFs semble indépendante de celle observée pour les récepteurs prolactiniques puisque Emane et al. (1986) ont démontré que ceux-ci augmentent brutalement au moment du déclenchement de la lactation. Enfin, il faut signaler que ces variations ont été analysées sans tenir compte de la modification de la densité du réseau membranaire que connaît la cellule mammaire au cours de la gestation et lors de l'installation de la lactation. C'est la concentration en récepteurs par unité de membrane (mg de protéine) qui a été considérée et non le nombre de récepteurs par cellule. En lactation, l'augmentation importante de la quantité de membrane extractible par cellule, compense probablement la diminution des récepteurs observée par unité de membrane.

La concentration élevée de récepteurs pour I'IGF $_{2}$ dans la glande mammaire (fig. 4) souligne l'importance de cette hormone dans le tissu mammaire. Aucun travail n'a été publié sur l'effet biologique de l'IGF ${ }_{2}$ sur la cellule mammaire. II est cependant tentant de spéculer qu'à l'instar de l'action de l'IGF $F_{2}$ dans d'autres modèles (Zapf et Froesch, 1985), I' $/ G F_{2}$ pourrait avoir un effet sur la différenciation des cellules épithéliales mammaires.

La caractérisation de récepteurs spécifiques pour les IGFs dans la glande mammaire renforce l'hypothèse de la médiation de l'action de la $\mathrm{GH}$ sur la lacta- 
tion par ces facteurs. Des travaux sur les actions biologiques des IGFs sur la multiplication et la différenciation cellulaire dans la glande mammaire - en cours au laboratoire - pourraient apporter un élément indispensable de réponse.

$12^{*}$ Réunion du groupe Développement I.N.R.A.

Montpellier, 28-30 mai 1986.

Recu en juillet 1987.

Accepté en septembre 1987.

Remerciements. - Les auteurs tiennent à remercier M. le Pr. Humbel (Zürich) pour les IGFs, le National Institute of Health (Dr. Raiti) pour la prolactine et I'hormone de croissance, Dr. Houdebine pour ses conseils et critiques et Mme Brugnolo pour la frappe du manuscrit.

\section{Références}

BOLANDER F. F., NICHOLAS K. R., VAN WYCK J. J., TOPPER Y. J., 1981. Insulin is essential for accumulation of casein mRNA in mouse mammary epithelial cells. Proc. nat. Acad. Sci. USA, 78, 5682-5684.

COWIE A. T., TINDAL J. S., 1960. Effects of hypophysectomy of the pregnant and lactating goat. Acta endocrinol., 35, suppl. 51, 679.

DENAMUR R., 1971. Hormonal control of lactogenesis. J. Dairy Res., 38, 237.

EMANE M. N., DELOUIS C., KELLY P. A., DJIANE J., 1986. Evolution of prolactin and placental lactogen receptors in ewes during pregnancy and lactation. Endocrinology, 118, 695-700.

ENRIGHT W. J., CHAPIN L. T., MOSELEY W. M., ZINW S. A., TUCKER H. A., 1986. Growth hormone-realising factor stimulates milk production and sustains growth hormone release in Holstein cowss. J. Dairy Sci., 69, 344-351.

FLIER J. S., USHER P., MOSES A. C., 1986. Monoclonal antibody to the type I insulin like growth factor $\left(I G F_{1}\right)$ receptor blocks $I_{1 G F}$ receptor mediated DNA synthesis: clarification of the mitogenic mechanisms of $\mathrm{IGF}_{1}$ and insulin in human skin fibroblasts. Proc. nat. Acad. Sci., 83, 664-668.

FURNALETTO R. W., DI CARLO J. N., 1984. Somatomedin C receptors and growth effect in human breast cells maintained in long term tissue culture. Cancer Res., 44, 2122-2128.

GERTLER A., COHEN N., MAOZ A., 1983. Human growth hormone but not ovine or bovine growth hormones exhibits galactopoietic prolactin-like activity in organ culture from bovine lactating mammary gland. Mol. cell. Endocr., 33, 169-182.

GERTLER A., ASHKENAZI A., MADAR Z., 1984. Binding sites of human growth hormone and ovine and bovine prolactins in the mammary gland and the liver of lactating dairy cow. Mol. cell. Endocr., 34, 51-53.

HART I. C., BINES J. A., JAMES S., MORANT S. V., 1985. The effect of injecting or infusing low doses of bovine growth hormone on milk yield, milk composition and the quantity of hor mone in the milk serum of cows. Nat. Proc., 40, 243-250.

HONEGGER A., HUMBEL R. E., 1986. Insulin-like growth factors I and II in fetal and adult bovine serum. Purification, primary structures and immunological cross-reactivities. J. biol. Chem., 261, 569-575.

HUNTER W. M., GREENWOOD F. C., 1962. Preparation of lodine 131 human growth hormone of high specific activity. Nature, 194, 495.

KEYS J. E., DJIANE J., 1987. Tissue distribution of somatotropic and lactogenic binding sites between mammary and liver tissues. J. Recept. Res. (soumis).

LOWRY O. H., ROSEBROUGH N. J., FARR A. L., ROMDALL R. J., 1951. Protein measurement with the folin phenol reagent. J. Biochem., 193, 265. 
PERDUE J. F., 1984. Chemistry, structure and function of insulin-like growth factors and their receptors : a review. Can. J. Biochem. Cell Biol., 62, 1237-1245.

RINDERKNECHT E., HUMBEL R. E., 1978. Primary structure of human insulin-like growth factor II. FEBS Letters, 89, 283-286.

ROSENFELD R. G., HINTZ R. L., 1986. Somatomedin receptors : structure, function and regulation. In P. M. Conn, The receptors. Vol. III, Acad. Press. Inc.

SCATCHARD G., 1949. The attractions of proteins for small molecules and ions. Ann. N.Y. Acad. Sci., 51, 660-672.

VAN WYK J. J., UNDERWOOD L. E., HINTZ R. L., CLEMMONS D. R., VOINA S. J., WEAVER R. P., 1974. The somatomedins : a family of insulin-like hormones under growth hormone control. Rec. Progr. Horm. Res., 30, 259-295.

VAN WYK J. J., GRAVES D. C., CASELLA S. J., JACOBS S., 1985. Evidence from monoclonal antibody studies that insulin stimulates deoxyribonucleic acid synthesis through the type I somatomedin receptor. J. clin. Endocrinol. Metab., 61, 639-643.

ZAPF J., FROESCH E. R., 1985. Insulin-like growth factors/somatomedin : structure secretion, biological actions and physiological role. Dans : Données récentes sur l'axe somatotrope: somatocrinine, somatomédines. $28 \mathrm{e}$ Jour. int. Henri-Pierre Klotz d'endocrinologie clinique, Paris, 31 mai-1er juin 1985, 2-20. 